\title{
Existence and Multiplicity of Solutions to a Boundary Value Problem for Impulsive Differential Equations
}

\author{
Chunyan He, ${ }^{1}$ Yongzhi Liao, ${ }^{2}$ and Yongkun $\mathrm{Li}^{1}$ \\ ${ }^{1}$ Department of Mathematics, Yunnan University, Kunming, Yunnan 650091, China \\ ${ }^{2}$ School of Mathematics and Computer Science, Panzhihua University, Panzhihua, Sichuan 617000, China \\ Correspondence should be addressed to Yongkun Li; yklie@ynu.edu.cn
}

Received 24 October 2012; Revised 20 December 2012; Accepted 21 December 2012

Academic Editor: Wan-Tong Li

Copyright (c) 2013 Chunyan He et al. This is an open access article distributed under the Creative Commons Attribution License, which permits unrestricted use, distribution, and reproduction in any medium, provided the original work is properly cited.

\begin{abstract}
We investigate the existence and multiplicity of solutions to a boundary value problem for impulsive differential equations. By using critical point theory, some criteria are obtained to guarantee that the impulsive problem has at least one solution, at least two solutions, and infinitely many solutions. Some examples are given to illustrate the effectiveness of our results.
\end{abstract}

\section{Introduction}

In this paper, we will investigate the existence and multiplicity of solutions to the boundary value problem for impulsive differential equations:

$$
\begin{gathered}
-\left(\rho(t)\left|u^{\prime}(t)\right|^{p-2} u^{\prime}(t)\right)^{\prime}+s(t)|u(t)|^{p-2} u(t) \\
=f(t, u(t)), \quad t \neq t_{i}, \text { a.e. } t \in[0, T], \\
\Delta\left(\rho\left(t_{i}\right)\left|u^{\prime}\left(t_{i}\right)\right|^{p-2} u^{\prime}\left(t_{i}\right)\right)=I_{i}\left(u\left(t_{i}\right)\right), \\
i=1,2, \ldots, k, \\
u(0)=u(T)=0,
\end{gathered}
$$

where $p \geq 2, \rho(t), s(t) \in L^{\infty}[0, T]$, with ess $\inf _{t \in[0, T]} \rho(t)>0$, ess $\inf _{t \in[0, T]} s(t)>0$, and $1 \leq \rho(t)<+\infty ; 0<s(t)<+\infty$; $0=t_{0}<t_{1} \cdots<t_{k}<t_{k+1}=T, \Delta\left(\rho\left(t_{i}\right)\left|u^{\prime}\left(t_{i}\right)\right|^{p-2} u^{\prime}\left(t_{i}\right)\right)=$ $\rho\left(t_{i}^{+}\right)\left|u^{\prime}\left(t_{i}^{+}\right)\right|^{p-2} u^{\prime}\left(t_{i}^{+}\right)-\rho\left(t_{i}^{-}\right)\left|u^{\prime}\left(t_{i}^{-}\right)\right|^{p-2} u^{\prime}\left(t_{i}^{-}\right), f:[0, T] \times$ $R \rightarrow R$ is continuous, $I_{i}: R \rightarrow R$ are continuous.

Recently, there have been many papers concerned with boundary value problems for impulsive differential equations. Impulsive effects exist widely in many evolution processes in which their states are changed abruptly at certain moments of time. The theory of impulsive differential systems has been developed by numerous mathematicians (see [1-6]).
Impulsive and periodic boundary value problems have been studied extensively in the literature. There have been many approaches to study periodic solutions of differential equations, such as the method of lower and upper solutions, fixed point theory, and coincidence degree theory (see [710]). However, the study of solutions for impulsive differential equations using variational method has received considerably less attention (see, [11-18]). Variational method is, to the best of our knowledge, novel and it may open a new approach to deal with nonlinear problems with some type of discontinuities such as impulses.

Teng and Zhang in [15] studied the existence of solutions to the boundary value problem for impulsive differential equations

$$
\begin{gathered}
-\left(\left|u^{\prime}(t)\right|^{p-2} u^{\prime}(t)\right)^{\prime}=f\left(t, u(t), u^{\prime}(t)\right), \\
t \neq t_{i}, \text { a.e. } t \in[0, T], \\
\Delta u^{\prime}\left(t_{i}\right)=I_{i}\left(u\left(t_{i}\right)\right), \quad i=1,2, \ldots, l, \\
u(0)=u(T)=0 .
\end{gathered}
$$

By using variational methods and iterative methods they showed that there exists a solution for problem (2).

In this paper, we will need the following conditions. 
(A) $F(t, u)$ is measurable in $t$ for every $u \in \mathbb{R}$ and continuously differentiable in $u$ for a.e. $t \in[0, T]$ and there exist $a \in C\left(\mathbb{R}^{+}, \mathbb{R}^{+}\right), b \in L^{1}\left(0, T ; \mathbb{R}^{+}\right)$such that

$$
|F(t, u)| \leq a(|u|) b(t), \quad|f(t, u)| \leq a(|u|) b(t),
$$

for all $u \in \mathbb{R}$ and a.e. $t \in[0, T]$, where $F(t, u)=$ $\int_{0}^{u} f(t, s) \mathrm{d} s$.

$\left(H_{0}\right)$ There exist constants $a, b>0$ and $r \in[0, p-1)$ such that

$$
|f(t, u)| \leq a+b|u|^{r}, \quad \text { for }(t, u) \in[0, T] \times R .
$$

$\left(H_{1}\right)$ There exist constants $c>0$ and $\tau \in[p,+\infty)$ such that

$$
|f(t, u)| \leq c\left(|u|^{\tau-1}+1\right), \quad \text { for }(t, u) \in[0, T] \times R .
$$

$\left(H_{2}\right)$ There exist constants $a_{i}, b_{i}>0$ and $r_{i} \in[0, p-1)(i=$ $1,2, \ldots, k)$ such that

$$
\left|I_{i}(u)\right| \leq a_{i}+b_{i}|u|^{r_{i}}, \quad \text { for } u \in R .
$$

$\left(H_{3}\right)$ There exist constants $\mu>p$ and $M>0$ such that

$$
0<\mu F(t, u) \leq u f(t, u), \quad \text { for } t \in[0, T],|u| \geq M \text {. }
$$

$\left(H_{4}\right) \lim _{u \rightarrow 0} f(t, u) /|u|^{p-1}=0$ uniformly, for $t \in[0, T]$ and $u \in R$.

$\left(H_{5}\right)$ there exist $\lambda>p$ and $\beta>\lambda-p$ such that

$\limsup _{|x| \rightarrow \infty} \frac{F(t, x)}{|x|^{\lambda}}<\infty \quad$ uniformly, for a.e. $t \in[0, T]$,

$$
\liminf _{|x| \rightarrow \infty} \frac{x f(t, x)-2 F(t, x)}{|x|^{\beta}}>0,
$$

uniformly for a.e. $t \in[0, T]$.

$\left(H_{6}\right)$ there exists $\zeta_{i j}>0$ such that

$2 \int_{0}^{t} I_{i}(s) \mathrm{d} s-I_{i}(t) t \geq 0 \quad \forall i \in\{1,2, \ldots, k\},|t| \geq \zeta_{i j}$

We recall some facts which will be used in the proof of our main results. It has been shown, for instance, in [19] that the set of all eigenvalues of the following problem:

$$
\begin{gathered}
-\left(\left|u^{\prime}(t)\right|^{p-2} u^{\prime}(t)\right)^{\prime}+\lambda|u(t)|^{p-2} u(t)=0, \\
t \neq t_{i}, t \in[0, T], \\
u(0)=u(T)=0
\end{gathered}
$$

is given by the sequence of positive numbers

$$
\lambda_{k}=(p-1)\left(\frac{k \pi_{p}}{T}\right)^{p}, \quad \text { for } k=1,2, \ldots,
$$

where

$$
\pi_{p}=2 \int_{0}^{1} \frac{1}{\left(1-s^{p}\right)^{1 / p}} \mathrm{~d} s .
$$

Each eigenvalue $\lambda_{k}$ is simple with the associated eigenfunction

$$
\varphi_{k}(x)=\sin _{p}\left(\frac{k \pi_{p} x}{T}\right), \quad \text { for } 0 \leq x \leq T .
$$

$X_{k}$ denotes the eigenspace associated to $\lambda_{k}$, then $W_{0}^{1, p}(0, T)=$ $\overline{\bigoplus_{i \in N} X_{i}}$.

An outline of this paper is given as follows. In the next section, we present some preliminaries including some basic knowledge and critical point theory. In Section 3, by using the critical point theory, we will establish some sufficient conditions for the existence of solutions of system (1). In Section 4, some examples are given to verify and support the theoretical findings.

\section{Preliminaries}

In this section, we recall some basic facts which will be used in the proofs of our main results. In order to apply the critical point theory, we make a variational structure. From this variational structure, we can reduce the problem of finding solutions of (1) to the one of seeking the critical points of a corresponding functional.

In [20], the Sobolev space $W_{0}^{1, p}(0, T)$ be the endowed with the norm

$$
\|u\|_{W_{0}^{1, p}}=\left(\int_{0}^{T}\left|u^{\prime}(t)\right|^{p} \mathrm{~d} t\right)^{1 / p}, \quad u \in W_{0}^{1, p}(0, T) .
$$

Throughout the paper, we also consider the norm

$$
\begin{array}{r}
\|u\|=\left(\int_{0}^{T}\left(\rho(t)\left|u^{\prime}(t)\right|^{p}+s(t)|u(t)|^{p}\right) \mathrm{d} t\right)^{1 / p}, \\
u \in W_{0}^{1, p}(0, T) .
\end{array}
$$

By Poincaré inequality:

$$
\int_{0}^{T}\left|u^{\prime}(t)\right|^{p} \mathrm{~d} t \geq C \int_{0}^{T}|u(t)|^{p} \mathrm{~d} t
$$

where $C=\lambda_{1}$ is precisely the largest $C>0$ for which the above inequality holds true. Then $\int_{0}^{T}\left|u^{\prime}(t)\right|^{p} \mathrm{~d} t-\lambda_{1} \int_{0}^{T}|u(t)|^{p} \mathrm{~d} t \geq$ 0 while it minimizes and equals to zero exactly on the ray generated by the first eigenfunction $\sin _{p}\left(\pi_{p} t / T\right)$.

Let us recall that

$$
|u|_{p}=\left(\int_{0}^{T}|u(t)|^{p} \mathrm{~d} t\right)^{1 / p}, \quad\|u\|_{\infty}=\max _{t \in[0, T]}|u(t)| .
$$

We denote by $|\cdot|_{p}$ the usual $L^{p}$-norm. The $n$-dimensional Lebesgue measure of a set $E \subseteq R^{n}$ is denoted by $|E|$. By the Sobolev embedding theorem, $W_{0}^{1, p}(0, T) \hookrightarrow L^{r}[0, T]$ 
continuously for $r \in[1,+\infty)$, and there exists $\gamma_{r}>0$ such that

$$
|u|_{r} \leq \gamma_{r}\|u\|, \quad \forall u \in W_{0}^{1, p}(0, T) .
$$

Lemma 1. There exist $C_{1}, C_{2}>0$ such that

$$
C_{1}\|u\|_{W_{0}^{1, p}} \leq\|u\| \leq C_{2}\|u\|_{W_{0}^{1, p}}, \quad \forall u \in W_{0}^{1, p}(0, T) .
$$

Proof. Since ess $\inf _{t \in[0, T]} \rho(t)>0$, ess $\inf _{t \in[0, T]} s(t)>0$, $1 \leq \rho(t)<+\infty$, and $0<s(t)<+\infty$, we have that ess $\inf _{t \in[0, T]} \rho(t):=m_{1} \geq 1$, ess inf $\operatorname{in}_{t \in[0, T]} s(t):=m_{2}>-\lambda_{1}$, where $\lambda_{1}$ is a positive number and that there exists $n_{1} \in(0,1)$ such that $-m_{2} \leq \lambda_{1}\left(1-n_{1}\right)$. Thus, by Poincaré inequality, we have

$$
\begin{aligned}
\left(1-n_{1}\right) \int_{0}^{T}\left|u^{\prime}(t)\right|^{p} \mathrm{~d} t & \geq\left(1-n_{1}\right) \lambda_{1} \int_{0}^{T}|u(t)|^{p} \mathrm{~d} t \\
& \geq-m_{2} \int_{0}^{T}|u(t)|^{p} \mathrm{~d} t,
\end{aligned}
$$

for all $u \in W_{0}^{1, p}(0, T)$. Thereby, for every $u \in W_{0}^{1, p}(0, T)$,

$$
\begin{aligned}
\|u\|^{p} & =\int_{0}^{T} \rho(t)\left|u^{\prime}(t)\right|^{p} \mathrm{~d} t+\int_{0}^{T} s(t)|u(t)|^{p} \mathrm{~d} t \\
& \geq m_{1} \int_{0}^{T}\left|u^{\prime}(t)\right|^{p} \mathrm{~d} t \\
& =m_{1}\|u\|_{W_{0}^{1, p}}^{p} \\
& =C_{1}^{p}\|u\|_{W_{0}^{1, p}}^{p} .
\end{aligned}
$$

On the other hand, by Poincaré inequality, one has

$$
\begin{aligned}
\|u\|^{p} & =\int_{0}^{T} \rho(t)\left|u^{\prime}(t)\right|^{p} \mathrm{~d} t+\int_{0}^{T} s(t)|u(t)|^{p} \mathrm{~d} t \\
& \leq\|\rho\|_{\infty} \int_{0}^{T}\left|u^{\prime}(t)\right|^{p} \mathrm{~d} t+\|s\|_{\infty} \int_{0}^{T}|u(t)|^{p} \mathrm{~d} t \\
& \leq\left(\|\rho\|_{\infty}+\frac{\|s\|_{\infty}}{\lambda_{1}}\right) \int_{0}^{T}\left|u^{\prime}(t)\right|^{p} \mathrm{~d} t \\
& =\left(\|\rho\|_{\infty}+\frac{\|s\|_{\infty}}{\lambda_{1}}\right)\|u\|_{W_{0}^{1, p}}^{p} \\
& =C_{2}^{p}\|u\|_{W_{0}^{1, p}}^{p}
\end{aligned}
$$
then

Take $C_{1}=\left(m_{1}+n_{1}-1\right)^{1 / p}, C_{2}=\left(\|\rho\|_{\infty}+\|s\|_{\infty} / \lambda_{1}\right)^{1 / p}$,

$$
C_{1}\|u\|_{W_{0}^{1, p}} \leq\|u\| \leq C_{2}\|u\|_{W_{0}^{1, p}} .
$$

The proof is complete.

Lemma 2. There exists $C_{3}>0$ such that if $u \in W_{0}^{1, p}(0, T)$, then

$$
\|u\|_{\infty} \leq C_{3}\|u\|
$$

Proof. If $u \in W_{0}^{1, p}(0, T)$, it follows from the mean value theorem that

$$
\frac{1}{T} \int_{0}^{T} u(s) \mathrm{d} s=u(\xi)
$$

for some $\xi \in(0, T)$. Hence, for $t \in[0, T]$, by Hölder inequality and Poincaré inequality

$$
\begin{aligned}
|u(t)| & =\left|u(\xi)+\int_{\xi}^{t} u^{\prime}(s) \mathrm{d} s\right| \\
& \leq|u(\xi)|+\int_{0}^{T}\left|u^{\prime}(s)\right| \mathrm{d} s \\
& \leq \frac{1}{T}\left|\int_{0}^{T} u(s) \mathrm{d} s\right|+T^{1 / q}\left(\int_{0}^{T}\left|u^{\prime}(t)\right|^{p} \mathrm{~d} s\right)^{1 / p} \\
& \leq\left(\left(\frac{C}{T}\right)^{1 / p}+T^{1 / q}\right)\left(\int_{0}^{T}\left|u^{\prime}(t)\right|^{p} \mathrm{~d} s\right)^{1 / p} \\
& \leq\left(\left(\frac{C}{T}\right)^{1 / p}+T^{1 / q}\right) \frac{1}{C_{1}}\|u\| .
\end{aligned}
$$

Hence, $\|u\|_{\infty} \leq\left((C / T)^{1 / p}+T^{1 / q}\right)\left(1 / C_{1}\right)\|u\|=C_{3}\|u\|$. The proof is complete.

Take $v \in W_{0}^{1, p}(0, T)$ and multiply the two sides of the equality

$$
-\left(\rho(t)\left|u^{\prime}(t)\right|^{p-2} u^{\prime}(t)\right)^{\prime}+s(t)|u(t)|^{p-2} u(t)=f(t, u(t))
$$

by $v$ and integrate it from 0 to $T$, we have

$$
\begin{gathered}
-\int_{0}^{T}\left(\rho(t)\left|u^{\prime}(t)\right|^{p-2} u^{\prime}(t)\right)^{\prime} v(t) \mathrm{d} t \\
\quad+\int_{0}^{T} s(t)|u(t)|^{p-2} u(t) v(t) \mathrm{d} t \\
=\int_{0}^{T} f(t, u(t)) v(t) \mathrm{d} t .
\end{gathered}
$$

Moreover,

$$
\begin{aligned}
& -\int_{0}^{T}\left(\rho(t)\left|u^{\prime}(t)\right|^{p-2} u^{\prime}(t)\right)^{\prime} v(t) \mathrm{d} t \\
& =-\sum_{i=0}^{k} \int_{t_{i}}^{t_{i+1}}\left(\rho(t)\left|u^{\prime}(t)\right|^{p-2} u^{\prime}(t)\right)^{\prime} v(t) \mathrm{d} t
\end{aligned}
$$




$$
\begin{aligned}
&=\sum_{i=0}^{k}\left(\rho\left(t_{i+1}^{-}\right)\left|u^{\prime}\left(t_{i+1}^{-}\right)\right|^{p-2} u^{\prime}\left(t_{i+1}^{-}\right) v\left(t_{i+1}^{-}\right)\right. \\
&-\rho\left(t_{i}^{+}\right)\left|u^{\prime}\left(t_{i}^{+}\right)\right|^{p-2} u^{\prime}\left(t_{i}^{+}\right) v\left(t_{i}^{+}\right) \\
&\left.-\int_{t_{i}}^{t_{i+1}} \rho(t)\left|u^{\prime}(t)\right|^{p-2} u^{\prime}(t) v^{\prime}(t) \mathrm{d} t\right) \\
&= \sum_{i=1}^{k}\left(\rho\left(t_{i}^{+}\right)\left|u^{\prime}\left(t_{i}^{+}\right)\right|^{p-2} u^{\prime}\left(t_{i}^{+}\right)-\rho\left(t_{i}^{-}\right)\right. \\
&\left.\times\left|u^{\prime}\left(t_{i}^{-}\right)\right|^{p-2} u^{\prime}\left(t_{i}^{-}\right)\right)-\rho(T)\left|u^{\prime}(T)\right|^{p-2} u^{\prime}(T) v(T) \\
&+\rho(0)\left|u^{\prime}(0)\right|^{p-2} u^{\prime}(0) v(0) \\
&+\int_{0}^{T} \rho(t)\left|u^{\prime}(t)\right|^{p-2} u^{\prime}(t) v^{\prime}(t) \mathrm{d} t \\
&= \sum_{i=1}^{k} I_{i}\left(u\left(t_{i}\right)\right) v\left(t_{i}\right)+\int_{0}^{T} \rho(t)\left|u^{\prime}(t)\right|^{p-2} u^{\prime}(t) v^{\prime}(t) \mathrm{d} t .
\end{aligned}
$$

Combining (28), we have

$$
\begin{aligned}
& \int_{0}^{T} \rho(t)\left|u^{\prime}(t)\right|^{p-2} u^{\prime}(t) v^{\prime}(t) \mathrm{d} t \\
& \quad+\int_{0}^{T} s(t)|u(t)|^{p-2} u(t) v(t) \mathrm{d} t+\sum_{i=1}^{k} I_{i}\left(u\left(t_{i}\right)\right) v\left(t_{i}\right) \\
& \quad=\int_{0}^{T} f(t, u(t)) v(t) \mathrm{d} t .
\end{aligned}
$$

Considering the above, we introduce the following concept solution for problem (1).

Definition 3. We say that a function $u \in W_{0}^{1, p}(0, T)$ is a solution of problem (1) if the identity

$$
\begin{aligned}
& \int_{0}^{T} \rho(t)\left|u^{\prime}(t)\right|^{p-2} u^{\prime}(t) v^{\prime}(t) \mathrm{d} t \\
& \quad+\int_{0}^{T} s(t)|u(t)|^{p-2} u(t) v(t) \mathrm{d} t+\sum_{i=1}^{k} I_{i}\left(u\left(t_{i}\right)\right) v\left(t_{i}\right) \\
& \quad=\int_{0}^{T} f(t, u(t)) v(t) \mathrm{d} t
\end{aligned}
$$

holds for any $v \in W_{0}^{1, p}(0, T)$.

Consider the functional $\varphi: W_{0}^{1, p}(0, T) \rightarrow R$ defined by

$$
\varphi(u)=\frac{1}{p}\|u\|^{p}+\sum_{i=1}^{k} \int_{0}^{u\left(t_{i}\right)} I_{i}(t) \mathrm{d} t-\int_{0}^{T} F(t, u(t)) \mathrm{d} t
$$

Using the continuity of $f$ and $I_{i}, i=1,2, \ldots, k$, one has that $\varphi \in C^{1}\left(W_{0}^{1, p}(0, T), R\right)$. For any $v \in W_{0}^{1, p}(0, T)$, we have

$$
\begin{aligned}
\varphi^{\prime}(u) v= & \int_{0}^{T} \rho(t)\left|u^{\prime}(t)\right|^{p-2} u^{\prime}(t) v^{\prime}(t) \mathrm{d} t \\
& +\int_{0}^{T} s(t)|u(t)|^{p-2} u(t) v(t) \mathrm{d} t \\
& +\sum_{i=1}^{k} I_{i}\left(u\left(t_{i}\right)\right) v\left(t_{i}\right)-\int_{0}^{T} f(t, u(t)) v(t) \mathrm{d} t
\end{aligned}
$$

Thus, the solutions of problem (1) are the corresponding critical points of $\varphi$.

Definition 4. Let $X$ be a normed space. A minimizing sequence for a function $\varphi: X \rightarrow(-\infty,+\infty)$ is a sequence $u_{k}$ such that $\varphi\left(u_{k}\right) \rightarrow \inf \varphi$ whenever $k \rightarrow+\infty$.

Definition 5. Let $X$ be a Banach space and let $\varphi: X \rightarrow$ $(-\infty,+\infty) . \varphi$ is said to be sequentially weakly lower semicontinuous if $\lim \inf _{k \rightarrow+\infty} \varphi\left(x_{k}\right) \geq \varphi(x)$ as $x_{k} \rightarrow x$ in $X$.

Definition 6. Let $E$ be a Banach space and let $c \in R$. For any sequence $\left\{u_{k}\right\}$ in $E$, if $\varphi\left(u_{k}\right)$ is bounded and $\varphi^{\prime}\left(u_{k}\right) \rightarrow 0$ as $k \rightarrow+\infty$ possesses a convergent subsequence, then we say that $\varphi$ satisfies the Palais-Smale condition (denoted by PS condition for short). We say that $\varphi$ satisfies the Palais-Smale condition at level $c$ (denoted by (PS) condition for short) if there exists a sequence $\left\{u_{k}\right\}$ in $E$ such that $\varphi\left(u_{k}\right) \rightarrow c$ and $\varphi^{\prime}\left(u_{k}\right) \rightarrow 0$ as $k \rightarrow+\infty$ implies that $c$ is a critical value of $\varphi$.

Definition 7. Let $E$ be a Banach space and let $\varphi: E \rightarrow$ $(-\infty,+\infty) . \varphi$ is said to be coercive if $\varphi(u) \rightarrow+\infty$ as $\|u\| \rightarrow$ $+\infty$.

Lemma 8 (see [12]). If $\varphi$ is sequentially weakly lower semicontinuous on a reflexive Banach space $X$ and has a bounded minimizing sequence, then $\varphi$ has a minimum on $X$.

Definition 9. Let $X$ be a real Banach space with a direct sum decomposition $X=X^{1} \oplus X^{2}$. The functional $\varphi \in C^{1}(X, R)$ is said to have a local linking at 0 , with respect to $\left(X^{1}, X^{2}\right)$, if, for some $r>0$,
(i) $\varphi(u) \geq 0, u \in X^{1},\|u\| \leq r$,
(ii) $\varphi(u) \leq 0, u \in X^{2},\|u\| \leq r$.

If $\varphi$ has a local linking at 0 , then 0 is critical point (the trivial one). Suppose, furthermore, that there are two sequences of finite dimensional subspaces $X_{1}^{1} \subset X_{2}^{1} \subset \cdots \subset$ $X^{1}$ and $X_{1}^{2} \subset X_{2}^{2} \subset \cdots \subset X^{2}$ such that

$$
X^{1}=\overline{\bigcup_{n} X_{n}^{1}}, \quad X^{2}=\overline{\bigcup_{n} X_{n}^{2}}
$$


Definition 10 (see [13, Definition 2.2]). Let $I \in C^{1}(X, \mathbb{R})$. The functional $I$ satisfies the $(C)^{*}$ condition if every sequence $\left(u_{\alpha_{n}}\right)$ such that $\alpha_{n}$ is admissible and

$$
\begin{gathered}
u_{\alpha_{n}} \in X_{\alpha_{n}}, \quad \sup \left|I\left(u_{\alpha_{n}}\right)\right|<\infty, \\
\left(1+\left\|u_{\alpha_{n}}\right\|\right) I^{\prime}\left(u_{\alpha_{n}}\right) \longrightarrow 0
\end{gathered}
$$

contains a subsequence which converges to a critical point of I.

Lemma 11 (see [13]). Suppose that $\varphi \in C^{1}(X, R)$ satisfies the following assumptions:

(1) $\varphi$ satisfies the $(C)^{*}$ condition,

(2) $\varphi$ has a local linking at 0 ,

(3) $\varphi$ maps bounded sets into bounded sets,

(4) for every $m \in N, \varphi(u) \rightarrow-\infty$ as $\|u\| \rightarrow+\infty, u \in$ $X_{m}^{1} \oplus X^{2}$.

Then $\varphi$ has at least two critical points.

Lemma 12 (see [14]). Let $E$ be a Banach space. Let $\varphi \in$ $C^{1}(X, R)$ be an even functional which satisfies the PS condition and $\varphi(0)=0$. If $E=V \oplus Y$, where $V$ is finite dimensional, and $\varphi$ satisfies the following conditions:

(1) there exist constants $\rho, \alpha$ such that $\left.\varphi\right|_{\partial B_{\rho} \cap Y} \geq \alpha$, where $B_{\rho}=\{x \in E:\|x\|<\rho\}$,

(2) for each finite-dimensional subspace $W \subset E$ there is $R=R(W)$ such that $\varphi(u) \leq 0$, for all $u \in W$ with $\|u\| \geq R$

Then $\varphi$ has an unbounded sequence of critical values.

\section{Existence of Periodic Solutions}

Theorem 13. Assume that $(A),\left(H_{0}\right)$, and $\left(H_{2}\right)$ are satisfied, then problem (1) has at least one solution.

Proof. Let $M_{1}=\max \left\{a_{1}, a_{2}, \ldots, a_{k}\right\}, M_{2}=\max \left\{b_{1}, b_{2}, \ldots\right.$, $\left.b_{k}\right\}$. By Lemma 2, we have

$$
\begin{aligned}
\varphi(u) & =\frac{1}{p}\|u\|^{p}+\sum_{i=1}^{k} \int_{0}^{u\left(t_{i}\right)} I_{i}(t) \mathrm{d} t-\int_{0}^{T} F(t, u(t)) \mathrm{d} t \\
& \geq \frac{1}{p}\|u\|^{p}-\sum_{i=1}^{k} \int_{0}^{u\left(t_{i}\right)}\left(a_{i}+b_{i}|u|^{r_{i}}\right) \mathrm{d} t
\end{aligned}
$$

$$
\begin{aligned}
& -\int_{0}^{T}\left(a|u|+b|u|^{r+1}\right) \mathrm{d} t \\
\geq & \frac{1}{p}\|u\|^{p}-k M_{1}\|u\|_{\infty} \\
& -M_{2} \sum_{i=1}^{k}\|u\|_{\infty}^{r_{i}+1}-a T\|u\|_{\infty}-b T\|u\|_{\infty}^{r+1} \\
\geq & \frac{1}{p}\|u\|^{p}-k M_{1} C_{3}\|u\| \\
& -M_{2} \sum_{i=1}^{k} C_{3}^{r_{i}+1}\|u\|^{r_{i}+1}-a T C_{3}\|u\|-b T C_{3}^{r+1}\|u\|^{r+1},
\end{aligned}
$$

for all $u \in W_{0}^{1, p}(0, T)$. This implies that $\lim _{\|u\| \rightarrow \infty} \varphi(u)=\infty$, and $\varphi$ is coercive.

On the other hand, we show that $\varphi$ is weakly lower semicontinuous. If $\left\{u_{k}\right\}_{k \in N} \subset W_{0}^{1, p}(0, T), u_{k} \rightarrow u$, then we have that $\left\{u_{k}\right\}_{k \in N}$ converges uniformly to $u$ on $[0, T]$ and $\liminf _{k \rightarrow \infty}\left\|u_{k}\right\| \geq\|u\|$. Thus

$$
\begin{aligned}
\liminf _{k \rightarrow \infty} \varphi\left(u_{k}\right)= & \liminf _{k \rightarrow \infty}\left(\frac{1}{p}\left\|u_{k}\right\|^{p}+\sum_{i=1}^{k} \int_{0}^{u_{k}\left(t_{i}\right)} I_{i}(t) \mathrm{d} t\right. \\
& \left.\quad-\int_{0}^{T} F\left(t, u_{k}(t)\right) \mathrm{d} t\right) \\
\geq & \frac{1}{p}\|u\|^{p}+\sum_{i=1}^{k} \int_{0}^{u\left(t_{i}\right)} I_{i}(t) \mathrm{d} t-\int_{0}^{T} F(t, u(t)) \mathrm{d} t \\
& =\varphi(u) .
\end{aligned}
$$

By Lemma $8, \varphi$ has a minimum point on $W_{0}^{1, p}(0, T)$, which is a critical point of $\varphi$. Hence, problem (1) has at least one solution. The proof is complete.

We readily have the following corollary.

Corollary 14. Assume that $(A),\left(H_{0}\right)$, and $\left(H_{2}\right)$ are satisfied and $f$ and the impulsive functions $I_{i}(i=1,2, \ldots, k)$ are bounded. Then problem (1) has at least one solution.

Lemma 15. Assume that $\left(H_{1}\right),\left(H_{2}\right)$, and $\left(H_{3}\right)$ are satisfied, then $\varphi(u)$ satisfies the PS condition.

Proof. Assume that $\left\{u_{n}\right\} \subset W_{0}^{1, p}(0, T)$ satisfies that $\varphi\left(u_{n}\right)$ is bounded and $\varphi^{\prime}\left(u_{n}\right) \rightarrow 0$ as $n \rightarrow+\infty$. We will prove that the sequence $\left\{u_{n}\right\}$ is bounded.

It follows from $\left(H_{1}\right),\left(H_{2}\right),\left(H_{3}\right)$, and Lemma 2 , we have

$$
\begin{aligned}
\mu \varphi\left(u_{n}\right)-\varphi^{\prime}\left(u_{n}\right) u_{n} \\
=\left(\frac{\mu}{p}-1\right)\left\|u_{n}\right\|^{p} \\
\quad+\sum_{i=1}^{k}\left[\mu \int_{0}^{u_{n}\left(t_{i}\right)} I_{i}(t) d t-I_{i}\left(u_{n}\left(t_{i}\right)\right) u_{n}\left(t_{i}\right)\right]
\end{aligned}
$$




$$
\begin{aligned}
& -\int_{0}^{T}\left(\mu F\left(t, u_{n}(t)\right)-f\left(t, u_{n}(t)\right) u_{n}(t)\right) \mathrm{d} t \\
\geq & \left(\frac{\mu}{p}-1\right)\left\|u_{n}\right\|^{p}-(\mu+1) \\
& \times\left(k M_{1} C_{3}\left\|u_{n}\right\|+M_{2} \sum_{i=1}^{k} C_{3}^{r_{i}+1}\left\|u_{n}\right\|^{r_{i}+1}\right) \\
& -\int_{\left\{\left|u_{n}\right| \leq u_{0}\right\}}\left(\mu F\left(t, u_{n}(t)\right)-f\left(t, u_{n}(t)\right) u_{n}(t)\right) \mathrm{d} t \\
\geq & \left(\frac{\mu}{p}-1\right)\left\|u_{n}\right\|^{p}-(\mu+1) \\
& \times\left(k M_{1} C_{3}\left\|u_{n}\right\|+M_{2} \sum_{i=1}^{k} C_{3}^{r_{i}+1}\left\|u_{n}\right\|^{r_{i}+1}\right)-C_{4} .
\end{aligned}
$$

Hence, $\left\{u_{n}\right\}$ is bounded in $W_{0}^{1, p}(0, T)$.

Since $W_{0}^{1, p}(0, T)$ is a reflexive Banach space, passing to a subsequence if necessary, we may assume that there is a $u \in$ $W_{0}^{1, p}(0, T)$ such that

$$
\begin{gathered}
u_{n} \rightarrow u \quad \text { in } W_{0}^{1, p}(0, T), \\
u_{n} \longrightarrow u \text { in } L^{p}[0, T],
\end{gathered}
$$

$\left\{u_{n}\right\}$ converges uniformly to $u$ on $[0, T]$.

Notice that

$$
\begin{aligned}
& \left(\varphi^{\prime}\left(u_{n}\right)-\varphi^{\prime}(u), u_{n}-u\right) \\
& =\int_{0}^{T} \rho(t)\left(\left|u_{n}^{\prime}(t)\right|^{p-2} u_{n}^{\prime}(t)-\left|u^{\prime}(t)\right|^{p-2} u^{\prime}(t)\right) \\
& \quad \times\left(u_{n}^{\prime}(t)-u^{\prime}(t)\right) \mathrm{d} t \\
& \quad+\int_{0}^{T} s(t)\left(\left|u_{n}(t)\right|^{p-2} u_{n}(t)-|u(t)|^{p-2} u(t)\right) \\
& \quad \times\left(u_{n}(t)-u(t)\right) \mathrm{d} t \\
& \quad \sum_{i=1}^{k}\left(I_{i}\left(u_{n}\left(t_{i}\right)\right)-I_{i}\left(u\left(t_{i}\right)\right)\right)\left(u_{n}\left(t_{i}\right)-u\left(t_{i}\right)\right) \\
& \quad-\int_{0}^{T}\left(f\left(t, u_{n}(t)\right)-f(t, u(t))\right)\left(u_{n}(t)-u(t)\right) \mathrm{d} t .
\end{aligned}
$$

Recalling the following well-known inequality: for any $x, y \in$ $R^{N}$

$$
\left(|x|^{p-2} x-|y|^{p-2} y\right)(x-y) \geq c_{p}|x-y|^{p}, \quad p \geq 2,
$$

for some constant $c_{p}$ (Lemma 4.2 in [21]) and using Schwarz inequality, we have

$$
\begin{gathered}
c_{p} \int_{0}^{T} \rho(t)\left|u_{n}^{\prime}(t)-u^{\prime}(t)\right|^{p} \mathrm{~d} t+c_{p} \int_{0}^{T} s(t)\left|u_{n}(t)-u(t)\right|^{p} \mathrm{~d} t \\
\leq\left\|\varphi^{\prime}\left(u_{n}\right)-\varphi^{\prime}(u)\right\|\left\|u_{n}-u\right\| \\
\quad-\sum_{i=1}^{k}\left(I_{i}\left(u_{n}\left(t_{i}\right)\right)-I_{i}\left(u\left(t_{i}\right)\right)\right)\left(u_{n}\left(t_{i}\right)-u\left(t_{i}\right)\right) \\
\quad+\int_{0}^{T}\left(f\left(t, u_{n}(t)\right)-f(t, u(t))\right)\left(u_{n}(t)-u(t)\right) \mathrm{d} t .
\end{gathered}
$$

Since

$$
\begin{aligned}
& \left(I_{i}\left(u_{n}\left(t_{i}\right)\right)-I_{i}\left(u\left(t_{i}\right)\right)\right)\left(u_{n}\left(t_{i}\right)-u\left(t_{i}\right)\right) \\
& \quad \leq\left(\left|I_{i}\left(u_{n}\left(t_{i}\right)\right)\right|+\left|I_{i}\left(u\left(t_{i}\right)\right)\right|\right)\left\|u_{n}-u\right\|_{\infty} .
\end{aligned}
$$

By the assumption $\left(H_{1}\right)$, we have

$$
\begin{aligned}
& \int_{0}^{T}\left(f\left(t, u_{n}(t)\right)-f(t, u(t))\right)\left(u_{n}(t)-u(t)\right) \mathrm{d} t \\
& \quad \leq \int_{0}^{T}\left(\left|f\left(t, u_{n}(t)\right)\right|+|f(t, u(t))|\right)\left|u_{n}(t)-u(t)\right| \mathrm{d} t \\
& \quad \leq c \int_{0}^{T}\left(2+\left|u_{n}\right|^{\tau-1}+|u|^{\tau-1}\right)\left|u_{n}(t)-u(t)\right| \mathrm{d} t \\
& \quad \leq C_{5}\left\|u_{n}-u\right\|_{\infty}\left(1+\left|u_{n}\right|_{\tau-1}^{\tau-1}+|u|_{\tau-1}^{\tau-1}\right) .
\end{aligned}
$$

From (39), it follows that $u_{n} \rightarrow u$ in $W_{0}^{1, p}(0, T)$. Thus, $\varphi(u)$ satisfies the PS condition. The proof is complete.

Lemma 16. Assume that $(A),\left(H_{2}\right),\left(H_{5}\right)$, and $\left(H_{6}\right)$ are satisfied, then $\varphi$ satisfies the $(C)^{*}$ condition.

Proof. Let $\left\{u_{\alpha_{n}}\right\}$ be a sequence in $W_{0}^{1, p}(0, T)$ such that $\alpha_{n}$ is admissible and

$$
\begin{gathered}
u_{\alpha_{n}} \in X_{\alpha_{n}}, \quad \sup \left|\varphi\left(u_{\alpha_{n}}\right)\right|<+\infty, \\
\left(1+\left\|u_{\alpha_{n}}\right\|\right) \varphi^{\prime}\left(u_{\alpha_{n}}\right) \longrightarrow 0,
\end{gathered}
$$

then there exist a constant $C_{4}>0$ such that

$$
\left|\varphi\left(u_{\alpha_{n}}\right)\right| \leq C_{4}, \quad\left(1+\left\|u_{\alpha_{n}}\right\|\right) \varphi^{\prime}\left(u_{\alpha_{n}}\right) \leq C_{4},
$$

for all large $n$. On the other hand, by $\left(H_{5}\right)$, there are constants $C_{5}>0$ and $\rho_{1}>0$ such that

$$
F(t, x) \leq C_{5}|x|^{\lambda}
$$

for all $|x| \geq \rho_{1}$ and a.e. $t \in[0, T]$. By (A) one has

$$
|F(t, x)| \leq \max _{s \in\left[0, \rho_{1}\right]} a(s) b(t)
$$


for all $|x| \leq \rho_{1}$ and a.e. $t \in[0, T]$. It follows from (47) and (48) that

$$
|F(t, x)| \leq \max _{s \in\left[0, \rho_{1}\right]} a(s) b(t)+C_{5}|x|^{\lambda} .
$$

From $\left(\mathrm{H}_{2}\right)$ and Lemma 2, we have that

$$
\begin{aligned}
\left|\sum_{i=1}^{k} \int_{0}^{\left|u\left(t_{i}\right)\right|} I_{i}(t) \mathrm{d} t\right| & \leq \sum_{i=1}^{k} \int_{0}^{\left|u\left(t_{i}\right)\right|}\left(a_{i}+b_{i}|t|^{r_{i}}\right) \mathrm{d} t \\
& \leq M_{1} k\|u\|_{\infty}+M_{2} \sum_{i=1}^{k}\|u\|_{\infty}^{r_{i}+1} \\
& \leq M_{1} k C_{3}\|u\|+M_{2} C_{3} \sum_{i=1}^{k}\|u\|^{r_{i}+1}
\end{aligned}
$$

for all $u \in W_{0}^{1, p}(0, T)$, where $M_{1}=\left\{a_{1}, a_{2}, \ldots, a_{k}\right\}, M_{2}=$ $\left\{b_{1}, b_{2}, \ldots, b_{k}\right\}$. Combining (49), (50), and Hölder's inequality, we have

$$
\begin{aligned}
\frac{1}{p}\left\|u_{\alpha_{n}}\right\|^{p}= & \varphi\left(u_{\alpha_{n}}\right)-\sum_{i=1}^{k} \int_{0}^{u_{\alpha_{n}}\left(t_{i}\right)} I_{i}(t) \mathrm{d} t \\
& +\int_{0}^{T} F\left(t, u_{\alpha_{n}}(t)\right) \mathrm{d} t \\
\leq & C_{4}+M_{1} k C_{3}\|u\|+M_{2} C_{3} \sum_{i=1}^{k}\|u\|^{r_{i}+1} \\
& +C_{5} \int_{0}^{T}\left|u_{\alpha_{n}}(t)\right|^{\lambda} \mathrm{d} t+\max _{s \in\left[0, \rho_{1}\right]} a(s) \int_{0}^{T} b(t) \mathrm{d} t \\
\leq & C_{4}+M_{1} k C_{3}\|u\|+M_{2} C_{3} \sum_{i=1}^{k}\|u\|^{r_{i}+1} \\
& +C_{5} \int_{0}^{T}\left|u_{\alpha_{n}}(t)\right|^{\lambda} \mathrm{d} t+C_{6},
\end{aligned}
$$

for all large $n$, where $C_{6}=\max _{s \in\left[0, \rho_{1}\right]} a(s) \int_{0}^{T} b(t) \mathrm{d} t$. On the other hand, by $\left(H_{5}\right)$, there exist $C_{7}>0$ and $\rho_{2}>0$ such that

$$
x f(t, x)-2 F(t, x) \geq C_{7}|x|^{\beta}
$$

for all $|x| \geq \rho_{2}$ and a.e. $t \in[0, T]$. By $(\mathrm{A})$,

$$
|x f(t, x)-2 F(t, x)| \leq C_{8} b(t)
$$

for all $|x| \leq \rho_{2}$ and a.e. $t \in[0, T]$, where $C_{8}=(2+$ $\left.\rho_{2}\right) \max _{s \in\left[0, \rho_{2}\right]} a(s)$. Combining (52) and (53), one has

$$
(f(t, x), x)-2 F(t, x) \geq C_{7}|x|^{\beta}-C_{7} \rho_{2}^{\beta}-C_{8} b(t),
$$

for all $x \in \mathbb{R}^{N}$ and a.e. $t \in[0, T]$. According to $\left(H_{6}\right)$, there exists $C_{9}>0$ such that

$$
2 \int_{0}^{t} I_{i j}(s) \mathrm{d} s-I_{i j}(t) t \geq-C_{9} \quad \forall i=1,2, \ldots, k, t \in \mathbb{R} .
$$

Thus by (46), (54), and (55), we obtain

$$
\begin{aligned}
(p+1) C_{4} \geq & p \varphi\left(u_{\alpha_{n}}\right)-\left\langle\varphi^{\prime}\left(u_{\alpha_{n}}\right), u_{\alpha_{n}}\right\rangle \\
= & p \sum_{i=1}^{k} \int_{0}^{u_{\alpha_{n}}\left(t_{i}\right)} I_{i}(t) \mathrm{d} t-u_{\alpha_{n}} I_{i}\left(u_{\alpha_{n}}\right) \\
& +\int_{0}^{T}\left[u_{\alpha_{n}}(t) f\left(t, u_{\alpha_{n}}(t)\right)-p F\left(t, u_{\alpha_{n}}(t)\right)\right] \mathrm{d} t \\
= & \sum_{i=1}^{k}\left(p \int_{0}^{u_{\alpha_{n}}\left(t_{i}\right)} I_{i}(t) \mathrm{d} t-I_{i}\left(u_{\alpha_{n}}\left(t_{i}\right)\right) u_{\alpha_{n}}\left(t_{i}\right)\right) \\
& +\int_{0}^{T}\left[\left(\nabla F\left(t, u_{\alpha_{n}}(t)\right), u_{\alpha_{n}}(t)\right)\right. \\
\geq & -k C_{9}+C_{7} \int_{0}^{T}\left|u_{\alpha_{n}}\right|^{\beta} \mathrm{d} t-C_{7} \rho_{2}^{\beta} T-C_{8} \\
& \times \int_{0}^{T} b(t) \mathrm{d} t,
\end{aligned}
$$

for all large $n$. From (56), $\int_{0}^{T}\left|u_{\alpha_{n}}\right|^{\beta} \mathrm{d} t$ is bounded. If $\beta>\lambda$, by Hölder's inequality, we have

$$
\int_{0}^{T}\left|u_{\alpha_{n}}\right|^{\lambda} \mathrm{d} t \leq T^{(\beta-\lambda) / \beta}\left(\int_{0}^{T}\left|u_{\alpha_{n}}\right|^{\beta} \mathrm{d} t\right)^{\lambda / \beta} .
$$

Since $\xi_{i j} \in[0,1)$ for all $i=1,2, \ldots, k$, by (51) and (57), $\left\{u_{\alpha_{n}}\right\}$ is bounded in $W_{0}^{1, p}(0, T)$. If $\beta \leq \lambda$, by Lemma 2 , we obtain

$$
\begin{aligned}
\int_{0}^{T}\left|u_{\alpha_{n}}(t)\right|^{\lambda} \mathrm{d} t & =\int_{0}^{T}\left|u_{\alpha_{n}}(t)\right|^{\beta}\left|u_{\alpha_{n}}(t)\right|^{\lambda-\beta} \mathrm{d} t \\
& \leq\left\|u_{\alpha_{n}}\right\|_{\infty}^{\lambda-\beta} \int_{0}^{T}\left|u_{\alpha_{n}}(t)\right|^{\beta} \mathrm{d} t \\
& \leq C_{1}^{\lambda-\beta}\left\|u_{\alpha_{n}}\right\|^{\lambda-\beta} \int_{0}^{T}\left|u_{\alpha_{n}}(t)\right|^{\beta} \mathrm{d} t .
\end{aligned}
$$

Since $\xi_{i j} \in[0,1), \lambda-\beta<2$, by (51) and (58), $\left\{u_{\alpha_{n}}\right\}$ is also bounded in $W_{0}^{1, p}(0, T)$. Hence, $\left\{u_{\alpha_{n}}\right\}$ is also bounded in $W_{0}^{1, p}(0, T)$. Going if necessary to a subsequence, we can assume that $u_{\alpha_{\alpha_{n}}} \rightarrow u$ in $W_{0}^{1, p}(0, T)$. As the same the proof of Lemma 15, Therefore, $u_{\alpha_{n}} \rightarrow u$ in $W_{0}^{1, p}(0, T)$. Hence $\varphi$ satisfies the $(C)^{*}$ condition.

Theorem 17. Assume that $(A),\left(H_{1}\right),\left(H_{2}\right),\left(H_{3}\right),\left(H_{5}\right)$, and $\left(H_{6}\right)$ are satisfied and the following conditions hold. 
$\left(H_{7}\right)$ There exist constants $\delta>0, K>0, \gamma>0$ such that

$\frac{\gamma}{p}|u|^{p}+\frac{2 k M_{1}}{T}|u| \leq F(t, u) \leq \frac{1}{2 p \gamma_{p}^{p}}|u|^{p}+K|u|^{p+1}$,

$$
\text { for } t \in[0, T],|u| \leq \delta
$$

$\left(H_{8}\right) I_{i}(u)(i=1,2, \ldots, k)$ is nondecreasing.

Then the problem (1) has at least two critical points.

Proof. Let $X_{n}^{1}=\operatorname{span}\left\{\lambda_{k+1}, \lambda_{k+2}, \ldots, \lambda_{k+n}\right\}, X_{n}^{2}=X^{2}=$ $\left(X^{1}\right)^{\perp}$ for $n \in N$. Then $X^{j}=\overline{\bigcup_{n \in N} X_{n}^{j}}, j=1,2$. If $u \in X^{1}$ one has $|u|_{p} \leq \gamma_{p}\|u\|$, and if $u \in X^{2}$, we have $\|u\|^{p} \leq \gamma|u|_{p}^{p}$.

Step 1. $\varphi$ has a local linking at 0 with respect to $\left(X^{1}, X^{2}\right)$.

For $u \in X^{1}$, using $\left(H_{7}\right)$ we have

$$
\sum_{i=1}^{k} \int_{0}^{u\left(t_{i}\right)} I_{i}(t) \mathrm{d} t \geq 0
$$

It follows from $\left(\mathrm{H}_{8}\right)$ that

$$
\begin{aligned}
\varphi(u) & =\frac{1}{p}\|u\|^{p}+\sum_{i=1}^{k} \int_{0}^{u\left(t_{i}\right)} I_{i}(t) \mathrm{d} t-\int_{0}^{T} F(t, u(t)) \mathrm{d} t \\
& \geq \frac{1}{p}\|u\|^{p}-\frac{1}{2 p \gamma_{p}^{p}}|u|_{p}^{p}-K|u|_{p+1}^{p+1} \\
& \geq \frac{1}{p}\|u\|^{p}-\frac{1}{2 p \gamma_{p}^{p}} \gamma_{p}^{p}\|u\|^{p}-K \gamma_{p+1}^{p+1}\|u\|^{p+1} \\
& =\frac{1}{2 p}\|u\|^{p}-K \gamma_{p+1}^{p+1}\|u\|^{p+1}
\end{aligned}
$$

Thus, $\varphi(u) \geq 0$ for $u \in X^{1}$ with $\|u\| \leq r_{1}$, where $r_{1}>0$ is small enough.

For $u \in X^{2}$, with $\|u\| \leq r_{2}:=\delta / C_{3}$, we have $|u| \leq \mid u \|_{\infty} \leq$ $C_{3}\|u\| \leq \delta$ since $\operatorname{dim} X^{2}=k<+\infty$. Thus, From $\left(H_{2}\right)$ and $\left(H_{8}\right)$ it follows that

$$
\begin{aligned}
\varphi(u)= & \frac{1}{p}\|u\|^{p}+\sum_{i=1}^{k} \int_{0}^{u\left(t_{i}\right)} I_{i}(t) \mathrm{d} t \\
& -\int_{0}^{T} F(t, u(t)) \mathrm{d} t \leq \frac{1}{p}\|u\|^{p} \\
& +\sum_{i=1}^{k} \int_{0}^{u\left(t_{i}\right)}\left(a_{i}+b_{i}|u|^{r_{i}}\right)
\end{aligned}
$$

$$
\begin{aligned}
& -\int_{0}^{T}\left(\frac{\gamma}{p}|u|^{p}+\frac{2 k M_{1}}{T}|u|\right) \mathrm{d} t \\
& \leq \frac{\gamma}{p}|u|_{p}^{p}+k M_{1}\|u\|_{\infty}+M_{2} \\
& \quad \times \sum_{i=1}^{k}\|u\|_{\infty}^{r_{i}+1}-\frac{\gamma}{p}|u|_{p}^{p}-2 k M_{1}\|u\|_{\infty} \\
& \leq-k M_{1}\|u\|_{\infty}+M_{2} \sum_{i=1}^{k}\|u\|_{\infty}^{r_{i}+1} \leq 0 .
\end{aligned}
$$

Take $r=\min \left\{r_{1}, r_{2}\right\}$. We know that $\varphi$ has a local linking at 0 with respect to $\left(X^{1}, X^{2}\right)$.

Step 2. $\varphi$ maps bounded sets into bounded sets.

Assume $\|u\| \leq R_{0}$, where $R_{0}$ is a constant. By $\left(H_{8}\right)$, one has

$$
\begin{aligned}
\varphi(u) \leq & \frac{1}{p}\|u\|^{p}+\sum_{i=1}^{k} \int_{0}^{u\left(t_{i}\right)} I_{i}(t) \mathrm{d} t \\
& +\int_{0}^{T}|F(t, u(t))| \mathrm{d} t \leq \frac{1}{p}\|u\|^{p}+k M_{1}\|u\|_{\infty} \\
& +M_{2} \sum_{i=1}^{k}\|u\|_{\infty}^{r_{i}+1}+\frac{1}{2 p}\|u\|^{p}+K \gamma_{p+1}^{p+1}\|u\|^{p+1} \\
\leq & \frac{3}{2 p}\|u\|^{p}+k M_{1} C_{3}\|u\|+M_{2} \\
& \times \sum_{i=1}^{k} C_{3}^{r_{i}+1}\|u\|^{r_{i}+1}+K \gamma_{p+1}^{p+1}\|u\|^{p+1} \\
\leq & \frac{3}{2 p}\left\|R_{0}\right\|^{p}+k M_{1} C_{3}\left\|R_{0}\right\|+M_{2} \\
& \times \sum_{i=1}^{k} C_{3}^{r_{i}+1}\left\|R_{0}\right\|^{r_{i}+1}+K \gamma_{p+1}^{p+1}\left\|R_{0}\right\|^{p+1}<\infty,
\end{aligned}
$$

which implies that $\varphi$ maps bounded sets into bounded sets.

Step 3. For every $m \in N, \varphi(u) \rightarrow-\infty$ as $\|u\| \rightarrow+\infty, u \in$ $X_{m}^{1} \oplus X^{2}$.

By $\left(H_{5}\right)$, for any $M_{0}>0$, there exists a constant $h\left(M_{0}\right)$ such that $F(t, u(t)) \geq M|u|^{p}-h\left(M_{0}\right)$ for all $(t, u) \in[0, T] \times R$. Since $\operatorname{dim}\left(X_{m}^{1} \oplus X^{2}\right)$ is of finite dimension, there exists $\gamma>0$ such that $\|u\|^{p} \leq \gamma|u|_{p}^{p}$ for all $u \in X_{m}^{1} \oplus X^{2}$, which implies

$$
\begin{aligned}
\varphi(u) \leq & \frac{1}{p}\|u\|^{p}+\sum_{i=1}^{k} \int_{0}^{u\left(t_{i}\right)} I_{i}(t) \mathrm{d} t \\
& -M|u|_{p}^{p}+h(M) T \\
\leq & \frac{1}{p}\|u\|^{p}+k M_{1}\|u\|_{\infty}+M_{2}
\end{aligned}
$$




$$
\begin{aligned}
& \times \sum_{i=1}^{k}\|u\|_{\infty}^{r_{i}+1}-\frac{M}{\gamma}\|u\|^{p}+h(M) T \\
\leq & \left(\frac{1}{p}-\frac{M}{\gamma}\right)\|u\|^{p}+k M_{1} C_{3}\|u\| \\
& +M_{2} \sum_{i=1}^{k} C_{3}^{r_{i}+1}\|u\|^{r_{i}+1}+h(M) T .
\end{aligned}
$$

Choosing $M>\gamma / p$, we have $\varphi(u) \rightarrow-\infty$ as $\|u\| \rightarrow$ $+\infty, u \in X_{m}^{1} \oplus X^{2}$.

Summing up the above, and by Lemma 16, $\varphi$ satisfies all the assumptions of Lemma 11. Hence by Lemma 11, problem (1) has at least one nontrivial solution. The proof of Theorem 17 is completed.

Theorem 18. Assume that $(A),\left(H_{1}\right),\left(H_{2}\right),\left(H_{3}\right)$, and $\left(H_{4}\right)$ are satisfied and the following conditions hold.

$$
\begin{aligned}
& \left(H_{9}\right) f(t, u)=-f(t,-u) \text { for }(t, u) \in[0, T] \times R . \\
& \left(H_{10}\right) I_{i}(u)=-I_{i}(-u)(i=1,2, \ldots, k) \text { and nondecreasing. }
\end{aligned}
$$

Then the problem (1) has an infinite number of nontrivial solutions.

Proof. $\varphi \in C^{1}\left(W_{0}^{1, p}(0, T), R\right)$, by $\left(H_{9}\right)$ and $\left(H_{10}\right), \varphi$ is an even functional and $\varphi(0)=0$.

First, we verify the condition (2) of Lemma 12.

$$
\begin{array}{cl}
\frac{\mu}{u} \leq \frac{f(t, u)}{F(t, u)}, & u \geq M_{0}, \\
\frac{\mu}{u} \geq \frac{f(t, u)}{F(t, u)}, & u \leq-M_{0} .
\end{array}
$$

Integrating (65) for $u$ from $\left[M_{0}, u\right]$ and $\left[u,-M_{0}\right]$, respectively, we have

$$
\begin{aligned}
& \mu \ln \frac{u}{M_{0}} \leq \ln \frac{F(t, u)}{F\left(t, u_{0}\right)}, \quad u \geq M_{0}, \\
& \mu \ln \frac{M_{0}}{-u} \geq \ln \frac{F(t, u)}{F\left(t,-u_{0}\right)}, \quad u \leq-M_{0} .
\end{aligned}
$$

That is,

$$
\begin{gathered}
F(t, u) \geq F\left(t, M_{0}\right)\left(\frac{u}{M_{0}}\right)^{\mu}, \quad u \geq M_{0}, \\
F(t, u) \geq F\left(t,-M_{0}\right)\left(\frac{-u}{M_{0}}\right)^{\mu}, \quad u \leq-M_{0} .
\end{gathered}
$$

Combining (67) and (68), we have

$$
F(t, u) \geq \alpha_{1}|u|^{\mu}, \quad|u| \geq M_{0},
$$

where

$$
\alpha_{1}=u_{0}^{-\mu} \min \left\{\min _{t \in[0, T]} F\left(t, M_{0}\right), \min _{t \in[0, T]} F\left(t,-M_{0}\right)\right\}>0 .
$$

On the other hand, by the continuity of $F(t, u), F(t, u)$ is bounded on $[0, T] \times\left[-M_{0}, M_{0}\right]$, there exists $K_{1}>0$ such that

$$
F(t, u) \geq-K_{1} \geq \alpha_{1}|u|^{\mu}-\alpha_{1}\left|M_{0}\right|^{\mu}-K_{1}, \quad|u| \leq M_{0} .
$$

Combining (69) and (71), we have

$$
F(t, u) \geq \alpha_{1}|u|^{\mu}-\alpha_{2}, \quad \forall(t, u) \in[0, T] \times R,
$$

where $\alpha_{2}=\alpha_{1}+K_{1}$.

For arbitrary finite-dimensional subspace $W \subset W_{0}^{1, p}(0$, $T$ ), and any $u \in W$, there exists $C_{10}=C_{10}(W)>0$ such that

$$
|u|_{\mu} \geq C_{10}\|u\|
$$

By $\left(\mathrm{H}_{2}\right),(72),(73)$, and Lemma 2, we have

$$
\begin{aligned}
\varphi(u)= & \frac{1}{p}\|u\|^{p}+\sum_{i=1}^{k} \int_{0}^{u\left(t_{i}\right)} I_{i}(t) \mathrm{d} t \\
& -\int_{0}^{T} F(t, u(t)) \mathrm{d} t \leq \frac{1}{p}\|u\|^{p} \\
& +\sum_{i=1}^{k} \int_{0}^{u\left(t_{i}\right)}\left(a_{i}+b_{i}|u|^{r_{i}}\right) \mathrm{d} t \\
& -\int_{0}^{T}\left(\alpha_{1} u^{\mu}-\alpha_{2}\right) \mathrm{d} t \leq \frac{1}{p}\|u\|^{p} \\
& +k M_{1}\|u\|_{\infty}+M_{2} \sum_{i=1}^{k}\|u\|_{\infty}^{r_{i}+1} \\
& -\alpha_{1}|u|_{\mu}^{\mu}+\alpha_{2} T \leq \frac{1}{p}\|u\|^{p}+k M_{1} C_{3}\|u\| \\
& +M_{2} \sum_{i=1}^{k} C_{3}^{r_{i}+1}\|u\|^{r_{i}+1}-\alpha_{1} C_{10}^{\mu}\|u\|^{\mu}+\alpha_{2} T,
\end{aligned}
$$

for every $u \in W$. This implies that $\varphi(u) \rightarrow-\infty$ as $u \in W$ and $\|u\| \rightarrow \infty$. So there exists $R(W)>0$ such that $\varphi \leq 0$ on for all $u \in W$ with $\|u\| \geq R$.

In the following, we verify the condition (1) of Lemma 12.

Let $V=X_{1} \oplus X_{2}, Y=\overline{\bigoplus_{i=3}^{\infty} X_{i}}$, then $W_{0}^{1, p}(0, T)=$ $V \oplus Y$ and $V$ is finite dimensional. Using $\left(H_{10}\right)$ we have

$$
\sum_{i=1}^{k} \int_{0}^{u\left(t_{i}\right)} I_{i}(t) \mathrm{d} t \geq 0
$$

By $\left(H_{3}\right)$ and $\left(H_{4}\right)$, we have

$$
\lim _{u \rightarrow 0} \frac{F(t, u)}{u^{p}}=0 .
$$

Hence, for $\epsilon=1 / 2 p \gamma_{p}^{p}$, there exists $\delta>0$ such that for every $u$ with $|u| \leq \delta$,

$$
|F(t, u)| \leq \frac{1}{2 p \gamma_{p}^{p}}|u|^{p}
$$


Hence, for any $u \in Y$ with $\|u\| \leq \delta / C_{3},\|u\|_{\infty} \leq \delta$, by (18), (54) and (55), we have

$$
\begin{aligned}
\varphi(u)= & \frac{1}{p}\|u\|^{p}+\sum_{i=1}^{k} \int_{0}^{u\left(t_{i}\right)} I_{i}(t) \mathrm{d} t \\
& -\int_{0}^{T} F(t, u(t)) \mathrm{d} t \geq \frac{1}{p}\|u\|^{p} \\
& -\int_{0}^{T} F(t, u(t)) \mathrm{d} t \geq \frac{1}{p}\|u\|^{p}-\frac{1}{2 p \gamma_{p}^{p}}|u|_{p}^{p} \\
\geq & \frac{1}{p}\|u\|^{p}-\frac{1}{2 p \gamma_{p}^{p}} \gamma_{p}^{p}\|u\|^{p}=\frac{1}{2 p}\|u\|^{p} .
\end{aligned}
$$

Take $\alpha=(1 / 2 p)\left(\delta^{p} / C_{3}^{p}\right), \rho=\delta / C_{3}$, then

$$
\varphi(u) \geq \alpha, \quad \forall u \in \partial B_{\rho} \cap Y .
$$

Hence, by Lemma 12 and Lemma $15, \varphi$ possesses infinite critical points, that is, problem (1) has infinite nontrivial solutions. The proof is complete.

\section{Example}

Example 19. Let $p=2, \rho(t)=1, s(t)=t, t_{1}=1 / 2$. Consider the boundary value problem

$$
\begin{gathered}
-u^{\prime \prime}(t)+t u(t)=t+\sqrt{u(t)}, \quad \text { a.e. } t \in[0,1], \\
u(0)=u(1)=0, \\
\Delta u^{\prime}\left(t_{1}\right)=u^{\prime}\left(t_{1}^{+}\right)-u^{\prime}\left(t_{1}^{-}\right)=1+\sqrt{u(t)} .
\end{gathered}
$$

It is easy to see that conditions $\left(\mathrm{H}_{0}\right)$ and $\left(\mathrm{H}_{2}\right)$ of Theorem 13 hold. According to Theorem 13, problem (80) has at least one solution.

Example 20. Let $p=2, \rho(t)=1, s(t)=2-t, t_{1}=1 / 3$. Consider the boundary value problem

$$
\begin{gathered}
-u^{\prime \prime}(t)+(2-t) u(t)=(u(t))^{2}, \quad \text { a.e. } t \in[0,1], \\
u(0)=u(1)=0, \\
\Delta u^{\prime}\left(t_{1}\right)=u^{\prime}\left(t_{1}^{+}\right)-u^{\prime}\left(t_{1}^{-}\right)=1+\sqrt{u(t)} .
\end{gathered}
$$

It is easy to check that all the conditions of Theorem 17 are satisfied. Thus, according to Theorem 17, problem (81) has at least two critical points.

Example 21. Let $p=3, \rho(t)=1+2 t, s(t)=2+t, t_{1}=1 / 2$. Consider the boundary value problem

$$
\begin{gathered}
-\left((1+2 t)\left|u^{\prime}(t)\right| u^{\prime}(t)\right)^{\prime}+(2+t) u(t)=(u(t))^{3}, \\
\text { a.e. } t \in[0,1], u(0)=u(1)=0, \\
\Delta u^{\prime}\left(t_{1}\right)=u^{\prime}\left(t_{1}^{+}\right)-u^{\prime}\left(t_{1}^{-}\right)=\sqrt[3]{u(t)} .
\end{gathered}
$$

It is easy to check that all the conditions of Theorem 18 are satisfied. Therefore, according to Theorem 18, problem (82) has infinite nontrivial solutions.

\section{Acknowledgment}

This work is supported by the National Natural Sciences Foundation of China under Grant no. 10971183.

\section{References}

[1] M. Benchohra, J. Henderson, and S. Ntouyas, Impulsive Differential Equations and Inclusions, vol. 2 of Contemporary Mathematics and Its Applications, Hindawi Publishing Corporation, New York, NY, USA, 2006.

[2] A. M. Samoilenko and N. A. Perestyuk, Impulsive Differential Equations, vol. 14 of World Scientific Series on Nonlinear Science. Series A: Monographs and Treatises, World Scientific Publishing, River Edge, NJ, USA, 1995.

[3] J. J. Nieto and R. Rodríguez-López, "Boundary value problems for a class of impulsive functional equations," Computers \& Mathematics with Applications, vol. 55, no. 12, pp. 2715-2731, 2008.

[4] W. M. Haddad, V. Chellaboina, and S. G. Nersesov, Impulsive and Hybrid Dynamical Systems: Stability, Dissipativity, and Control, Princeton Series in Applied Mathematics, Princeton University Press, Princeton, NJ, USA, 2006.

[5] Z. Luo and J. J. Nieto, "New results for the periodic boundary value problem for impulsive integro-differential equations," Nonlinear Analysis: Theory, Methods \& Applications, vol. 70, no. 6, pp. 2248-2260, 2009.

[6] J. Chu and J. J. Nieto, "Impulsive periodic solutions of firstorder singular differential equations," Bulletin of the London Mathematical Society, vol. 40, no. 1, pp. 143-150, 2008.

[7] E. K. Lee and Y.-H. Lee, "Multiple positive solutions of singular two point boundary value problems for second order impulsive differential equations," Applied Mathematics and Computation, vol. 158, no. 3, pp. 745-759, 2004.

[8] M. Yao, A. Zhao, and J. Yan, "Periodic boundary value problems of second-order impulsive differential equations," Nonlinear Analysis: Theory, Methods \& Applications, vol. 70, no. 1, pp. 262273, 2009.

[9] X. Lin and D. Jiang, "Multiple positive solutions of Dirichlet boundary value problems for second order impulsive differential equations," Journal of Mathematical Analysis and Applications, vol. 321, no. 2, pp. 501-514, 2006.

[10] Y. Tian and W. Ge, "Multiple positive solutions for a second order Sturm-Liouville boundary value problem with a $p$ Laplacian via variational methods," The Rocky Mountain Journal of Mathematics, vol. 39, no. 1, pp. 325-342, 2009.

[11] J. Zhou and Y. Li, "Existence and multiplicity of solutions for some Dirichlet problems with impulsive effects," Nonlinear Analysis: Theory, Methods \& Applications, vol. 71, no. 7-8, pp. 2856-2865, 2009.

[12] J. Mawhin and M. Willem, Critical Point Theory and Hamiltonian Systems, vol. 74 of Applied Mathematical Sciences, Springer, New York, NY, USA, 1989.

[13] S. Luan and A. Mao, "Periodic solutions for a class of nonautonomous Hamiltonian systems," Nonlinear Analysis: Theory, Methods \& Applications, vol. 61, no. 8, pp. 1413-1426, 2005.

[14] P. H. Rabinowitz, Minimax Methods in Critical Point Theory with Applications to Differential Equations, vol. 65 of CBMS Regional Conference Series in Mathematics, American Mathematical Society, Providence, RI, USA, 1986. 
[15] K. Teng and C. Zhang, "Existence of solution to boundary value problem for impulsive differential equations," Nonlinear Analysis: Real World Applications, vol. 11, no. 5, pp. 4431-4441, 2010.

[16] J. J. Nieto and D. O'Regan, "Variational approach to impulsive differential equations," Nonlinear Analysis: Real World Applications, vol. 10, no. 2, pp. 680-690, 2009.

[17] Y. Tian and W. Ge, "Applications of variational methods to boundary-value problem for impulsive differential equations," Proceedings of the Edinburgh Mathematical Society. Series II, vol. 51, no. 2, pp. 509-527, 2008.

[18] L. Bai and B. Dai, "Existence and multiplicity of solutions for an impulsive boundary value problem with a parameter via critical point theory," Mathematical and Computer Modelling, vol. 53, no. 9-10, pp. 1844-1855, 2011.

[19] M. del Pino, M. Elgueta, and R. Manásevich, "A homotopic deformation along $p$ of a Leray-Schauder degree result and existence for $\left(\left|u^{\prime}\right|^{p-2} u^{\prime}\right)^{\prime}+f(t, u)=0, u(0)=u(T)=0, p>1$," Journal of Differential Equations, vol. 80, no. 1, pp. 1-13, 1989.

[20] W. D. Lu, Variational Methods in Differential Equations, Scientific Publishing House, Beijing, China, 2002.

[21] P. Lindqvist, "On the equation $\operatorname{div}\left(|\nabla u|^{p-2} \nabla u\right)+\lambda|u|^{p-2} u=0$," Proceedings of the American Mathematical Society, vol. 109, no. 1, pp. 157-164, 1990. 


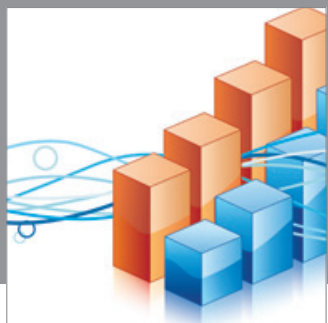

Advances in

Operations Research

mansans

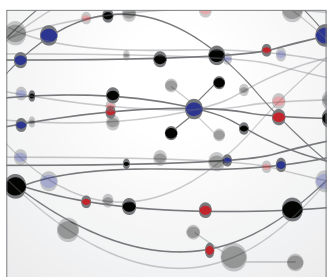

The Scientific World Journal
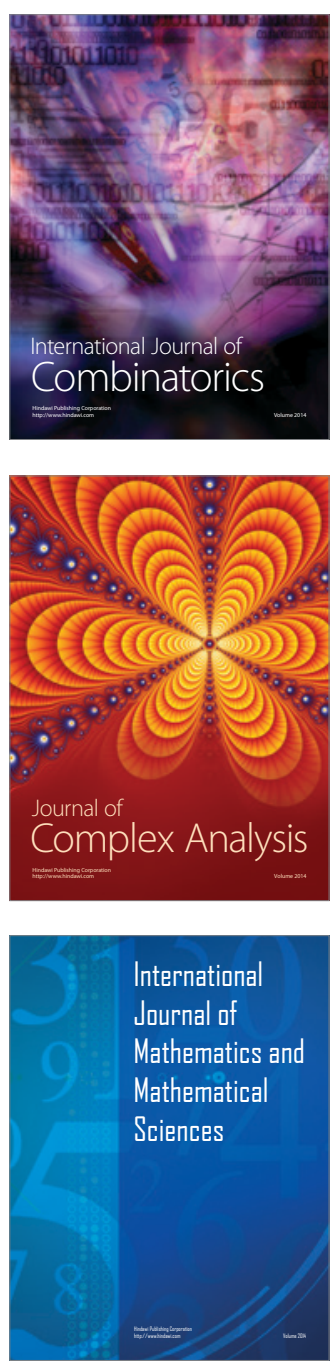
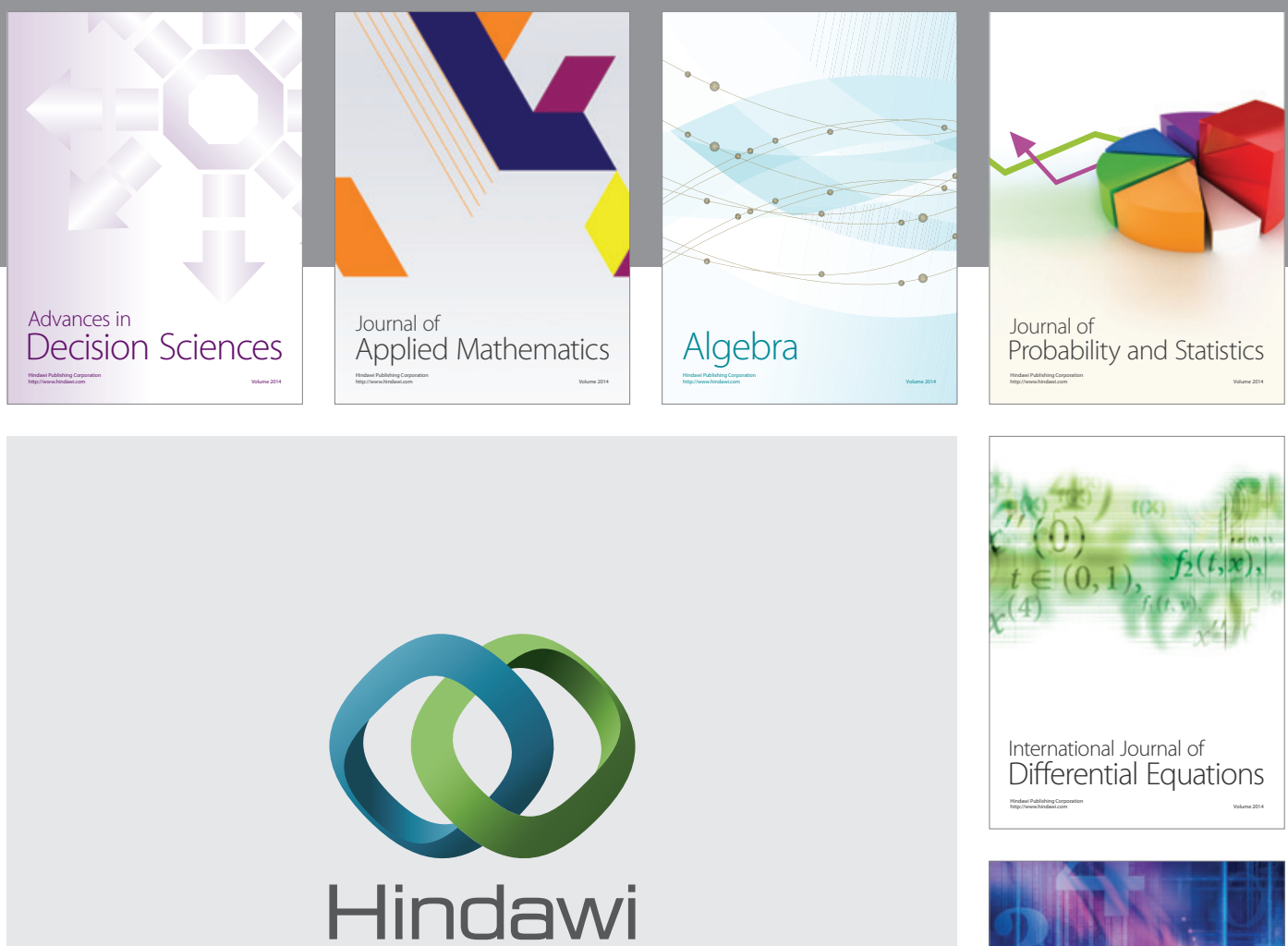

Submit your manuscripts at http://www.hindawi.com
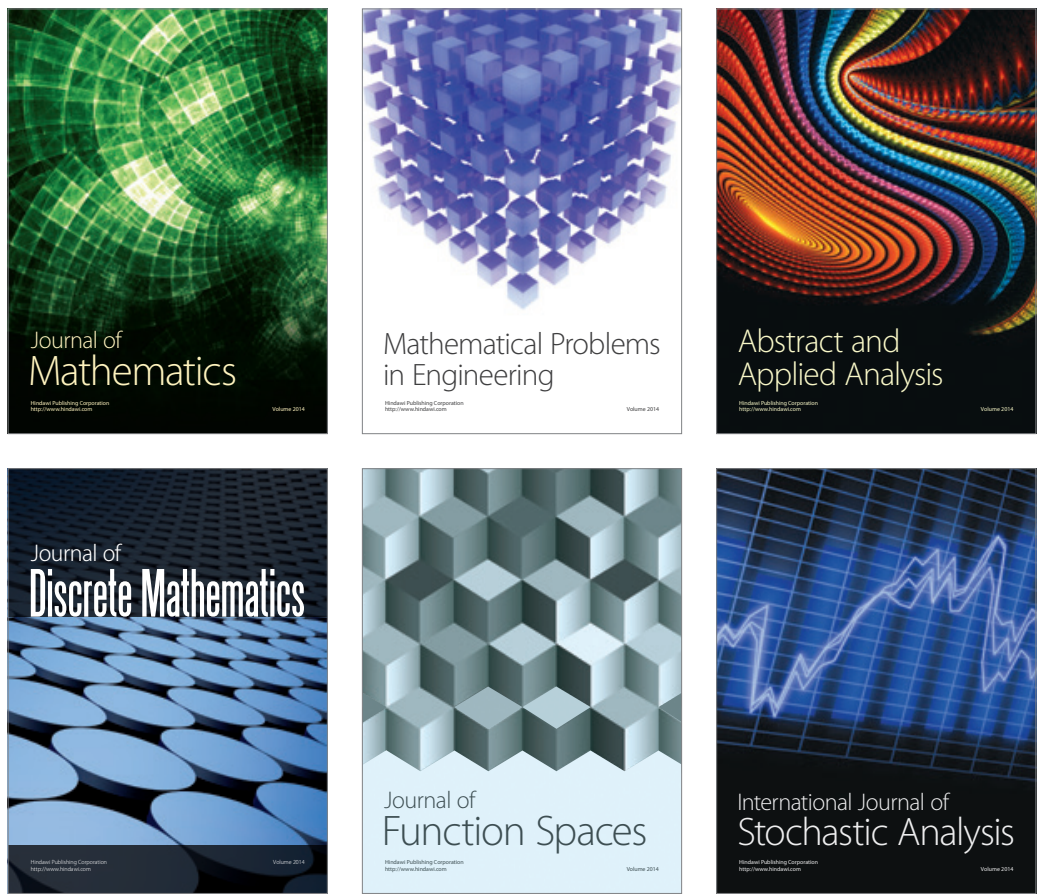

Journal of

Function Spaces

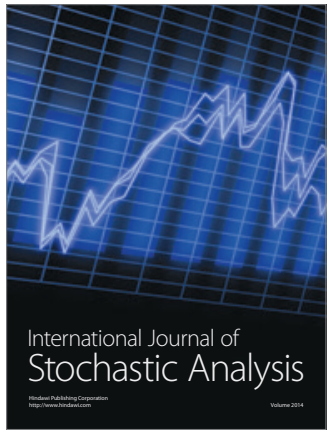

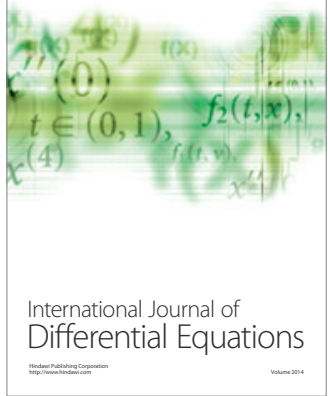
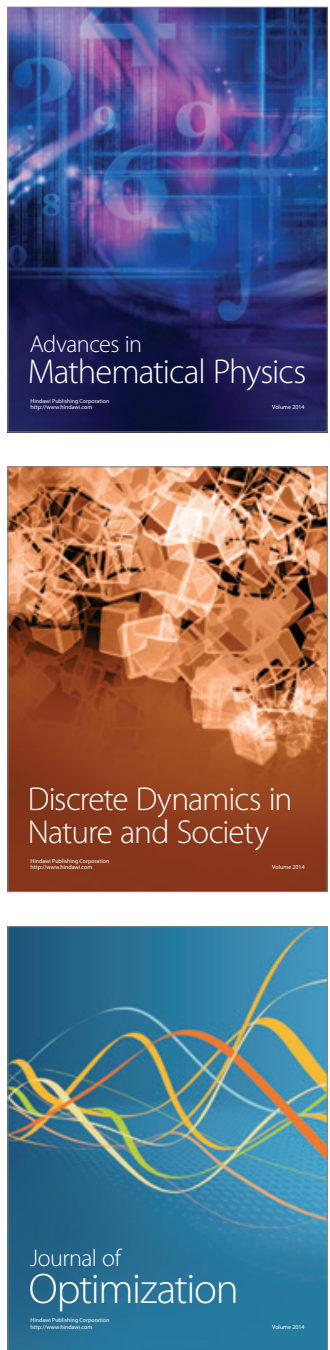